\title{
SABERES Y PRÁCTICAS DEL CUIDADO DE LA SALUD EN NIÑOS INDÍGENAS: UNA PERSPECTIVA DESDE LA ETNIA GUAMBIANA
}

\author{
Carmita Cepeda Chamorro ${ }^{1}$, Vanesa Flórez Meza ${ }^{2}$, Martha Lucía Vásquez Truisi ${ }^{2}$
}

\section{Resumen}

Introducción: La cultura es imprescindible cuando se quieren abordar las prácticas y saberes de una comunidad en torno a su perspectiva de salud y enfermedad para ofrecer un cuidado culturalmente congruente. Las acciones de la enfermería deben sustentarse en la adquisición de competencias culturales para obtener resultados buenos y eficaces. Objetivo: Comprender los saberes y prácticas para la prevención y tratamiento de problemas respiratorios y gastrointestinales en niños guambianos menores de un año. Metodología: Se realizó una etnografía focalizada utilizando entrevistas en profundidad. Se contó con la participación de: un médico tradicional, tres parteras (dos de ellas médicas tradicionales) y nueve madres de niños menores de un año con antecedentes de IRA y EDA. Hallazgos: Siete dominios emergieron, entre ellos: El susto, el manejo energético del niño y el poder curativo de las plantas, los cuales mostraron que la cosmovisión de los participantes se orienta hacia conservar la armonía entre ellos y la madre naturaleza. Conclusión: La cultura guambiana considera que la enfermedad se origina en una pérdida de la armonía de la persona con la naturaleza, con el espíritu y con su cuerpo. En consecuencia, los indígenas adoptan estilos de vida y costumbres en pro de la preservación de dicho equilibrio y, en general, del equilibrio entre el individuo y el ambiente que lo rodea.

Palabras clave: cuidado cultural, competencia cultural, enfermería transcultural, cuidado de enfermería.

${ }^{1}$ Enfermera Asistencial Hospital San José de Popayán. Docente catedrática Universidad del Cauca, Popayán, Colombia.

${ }^{2}$ Enfermera, Coordinadora Unidad de Cuidado Intensivo Neonatal Clínica la Estancia, Popayán. Docente catedrática Universidad del Cauca, Popayán, Colombia.

${ }^{3}$ Profesora Titular Jubilada, Universidad del Valle, Cali, Colombia. 


\section{KNOWLEDGE AND PRACTICES OF HEALTH CARE IN INDIGENOUS CHILDREN: A GUAMBIANA ETNIA PERSPECTIVE}

Carmita Cepeda Chamorro ${ }^{1}$, Vanesa Flórez Meza ${ }^{2}$, Martha Lucia Vásquez Truisi ${ }^{2}$

\section{Abstract}

Introduction. Culture is essential to approach the practices and knowledge of a community taking into account its perspective on health and disease, in order to provide a culturally consistent care. The nurse performance should have its foundation in the acquisition of cultural competences to obtain good and effective results. Objective. To understand the knowledge and practices performed for the prevention and treatment of respiratory and gastrointestinal issues in Guambian children under one year. Methodology. A focused ethnography was conducted using in-depth interviews. A traditional doctor was part of the research, three midwives (two of them traditional doctors) and nine mothers of children under one-year age with a history of IRA and EDA. Findings. Seven domains emerged, among them: The scare, the child energetic management and the healing power of plants, which showed that participants world-view is oriented towards preserving the harmony between them and Mother Nature. Conclusion. The Guambian culture considers that a disease is originated when the person losses harmony with nature, the spirit and the body. Consequently, indigenous people adopt lifestyles and customs which seeks the preservation of such balance and, in general, between the individual and the environment. 


\section{CONHECIMENTO E PRÁTICAS DA ATENÇÃO À SAÚDE DAS CRIANÇAS INDÍGENAS: UMA PERSPECTIVA DA ETNIA MISAK}

Carmita Cepeda Chamorro ${ }^{1}$, Vanesa Flórez Meza ${ }^{2}$, Martha Lucia Vásquez Truisi ${ }^{2}$

\section{Resumo}

Introdução. A cultura é essencial quando abordar as práticas e conhecimento de uma comunidade em torno de sua perspectiva de saúde e doença para oferecer atendimento culturalmente consistente. As ações do enfermeiro devem basear-se na aquisição de competências culturais para obter resultados apropriados e eficazes. Objetivo. Compreender os conhecimentos e práticas para a prevenção e tratamento de problemas respiratórios e gastrointestinais em crianças de indígenas misak com menos de um ano de idade. Metodologia. Uma etnografia focada foi realizada usando entrevistas em profundidade. Participaram um médico tradicional, três parteiras (duas delas médicos tradicionais) e nove mães de crianças menores de um ano com história de IRA e EDA. Resultados. Sete domínios surgiram, entre eles: o susto, o manejo energético da criança e o poder de cura das plantas, os quais mostraram que a cosmovisão dos participantes é orientada a preservar a harmonia entre a pessoa e a mãe natureza. Conclusão. A cultura Misak considera que a doença se origina em uma perda da harmonia da pessoa com a natureza, com o espírito e com o seu corpo. Consequentemente, os povos indígenas adotam estilos de vida e costumes em favor da preservação deste equilíbrio e, em geral, do equilíbrio entre o indivíduo e o meio ambiente que o rodeia. 


\section{Introducción}

"Se cuida el patrimonio de la humanidad cuando se aprende a cuidar, a respetar y a reconocer a los seres humanos de cada cultura, incluyendo la propia".

La cultura es transversal a cualquier tema humano porque ella construye y determina el conocimiento, las creencias, el arte, la moral, el derecho, las costumbres $y$, en general, todo lo que una persona adquiere como miembro de la sociedad en la que ha nacido y crecido. La salud no puede ser la excepción, pues también ella es una construcción social en la que intervienen los sentidos que se atribuyen a enfermedad, vida, muerte y que, por tanto, orientan las decisiones personales y comunitarias sobre cómo cultivarla y preservarla (1).

La atención a las infecciones respiratorias agudas (IRA) y gastrointestinales son las causas principales de consulta pediátrica, en particular -y muy intensamente- en niños y niñas menores de un año. Tanto atención primaria como los servicios de urgencia en la ESE MAMA DOMINGA del resguardo indígena de Guambía representan dos de los principales problemas de salud pública en este contexto, debido a la alta morbilidad que generan y su consecuente repercusión socioeconómica. Estos asuntos se ganan complejidad por las tensiones entre la mirada convencional-institucional acerca de la salud y los saberes y prácticas culturalmente sustentados y adoptados por los miembros de esta cultura.

180 Comprender la perspectiva de los fenómenos culturales que afectan a los niños y el razonamiento usado para acudir a los remedios tradicionales por parte de esta comunidad indígena en relación con las patologías mencionadas, es algo fundamental para el equipo de salud, pues las principales barreras que generan atención tardía o desatención se asocian con el hecho de que los usuarios indígenas no sienten que sus saberes sean reconocidos en la práctica médica institucional (2).

El presente estudio cualitativo se planteó desde la perspectiva metodológica de la etnografía focalizada para indagar sobre las prácticas y saberes de la cultura guambiana en el cuidado de los niños menores de un año con infecciones respiratorias y gastrointestinales, dimensionar su valor $y$ proveer al personal de salud información que le permita ofrecer un cuidado culturalmente congruente. Con este objetivo, se describen a continuación los principales ítems para el desarrollo de la investigación, donde los resultados se presentan organizados en siete dominios o categorías y se agregan dos temas, que son síntesis del conjunto de temas expuestos.

\section{Enfermería transcultural}

La enfermería transcultural se centra en el estudio comparativo y el análisis de la cultura de las personas con diferentes prácticas, creencias y valores de la salud-enfermedad. Esta área de la enfermería considera que hay cuidados culturales diversos y universales y ello está condicionado por las creencias de las personas de diferentes culturas, así que pueden informar y determinar qué tipo de cuidados desean o necesitan recibir de sus cuidadores profesionales. Puede 
definirse también como el conjunto de actividades profesionales destinadas al cuidado, promoción, mantenimiento o restablecimiento de la salud óptima, tanto para la persona como para la comunidad (3). Leininger (4) definió la enfermería transcultural como un área de la enfermería que se centra en el estudio comparativo y en el análisis de las diferentes culturas y subculturas del mundo, con respecto a los valores sobre los cuidados, la expresión y las creencias de la salud y la enfermedad, y el modelo de conducta. Esta autora afirma que la cultura y el cuidado son los medios más amplios para conceptualizar y entender a las personas y que este saber es imprescindible para la formación y la práctica en enfermería (5); además, defiende que así como la enfermería es significativa para los pacientes y para los profesionales de enfermería, el saber de la enfermería transcultural y sus competencias serán imprescindibles para orientar de manera congruente la cultura las decisiones y las acciones de los profesionales de enfermería, de modo que se obtengan resultados buenos y eficaces. Por lo tanto, hoy en día la cultura necesita estar presente en toda reflexión teórica sobre el cuidado, pues no se pretende que los cuidados de una cultura sean válidos para todas las demás: cada persona, grupo o subgrupo tiene sus prácticas, sus creencias, sus valores y sus tradiciones (3).

Para el estudio del cuidado, desde la perspectiva cultural, es imperiosa la necesidad de adquirir las competencias culturales, entendidas como ser sensible a los asuntos relacionados con la cultura, la raza, el género, la orientación sexual, la situación social y económica. Esto implica no sólo comprender las necesidades de los otros, sino tener las habilidades de intervenir apropiadamente en el cuidado de las personas. Para el desarrollo de dichas habilidades se requiere valorar, aceptar y respetar la diversidad; tener la capacidad, compromiso y sistemas en funcionamiento para la autoevaluación cultural; estar consciente de las dinámicas que ocurren cuando las culturas se comunican y adaptarse para crear espacios a la diversidad (6).

\section{Cultura guambiana}

La etnia guambiana está localizada en la parte occidental de la cordillera central, a 3000 metros de altura sobre el nivel del mar, en el departamento del Cauca, aunque existen algunos asentamientos en el municipio de La Plata, en el Huila. Los guambianos habitan los municipios de Silvia y Jambaló; unos pocos viven en los municipios de Totoró, Caldono y Toribío. Sus tierras están regadas por numerosos ríos y riachuelos, entre ellos, el río Piendamó, que atraviesa el resguardo de Guambia y es la principal corriente fluvial de esta región.

El territorio se divide en dos zonas: la primera, más baja y pendiente, es donde se produce maíz y trigo. Productos como la papa, la cebolla, el ulluco y el ajo se producen en la parte más alta y fría de esta primera zona. La otra zona la conforman las tierras paramunas, donde no se cultiva debido a la humedad, el frío y la fuerza de los vientos. Para los guambianos, esta zona está llena de misterio, ya que es el hábitat de fuerzas y espíritus sobrenaturales. Los datos oficiales revelan que el número de habitantes está por 
el orden de las 20782 personas, es decir, el 3\% de la población indígena nacional. Ocupan una extensión 18521 hectáreas, en un territorio caracterizado por su alto nivel de precipitaciones (7).

El indígena guambiano es de estatura promedio, de complexión vigorosa, frente estrecha, y cabeza, manos y pies pequeños. Su boca es grande, sus dientes, sanos y parejos, sus ojos, pequeños y su rostro, lampiño (8). Hablan la lengua guambiana, wampi-misamera-wam o "lengua de los hombres guambianos". Según expertos (8), junto con el Páez y el kamsá, son lenguas aisladas y de dudosa clasificación.

Para que el equipo de salud pueda fomentar las prácticas culturales en torno al cuidado de los niños menores de un año de la cultura indígena guambiana con las patologías referidas, es importante comprender la perspectiva de los fenómenos culturales que afectan a los niños y el razonamiento usado para acudir a los remedios tradicionales. Entender cuáles remedios son usados por los padres es importante para que el profesional de enfermería pueda ofrecer un cuidado seguro y apropiado y tenga mayores posibilidades de controlar cualquier factor de riesgo que pueda estar presente cuando alguna de estas prácticas culturales muestre contraindicaciones al cuidado concebido desde los conocimientos de la enfermería.

\section{Metodología}

Se realizó un estudio cualitativo, utilizando como método la etnografía foca-

lizada. La investigación se centró en un problema y contexto específico: los cui- dados que brindan las madres, parteras y médicos tradicionales de la cultura indígena guambiana a los niños menores de un año con problemas respiratorios y gastrointestinales. Se contó con el permiso del Cabildo Indígena para el desarrollo del estudio y con la aprobación del comité de ética de la Facultad de Salud de la Universidad del Valle; además del consentimiento informado de cada uno de los participantes en el grupo focal, donde se especificó la participación voluntaria y el propósito del estudio.

Dado su carácter etnográfico, el estudio se orientó en la clasificación sistemática (dominios) de las prácticas y saberes a través de las expresiones émicas, como lo propone en la etnoenfermeria Leininger(5) para el análisis de los datos. En ese sentido, los criterios de inclusión al estudio fueron ser madre de la cultura guambiana cuyo hijo menor de un año hubiera tenido infección respiratoria o gastrointestinal y que hubiera sido atendido por esa causa en la ESE Mama Dominga durante el tiempo en que se recolectó la información (abril y diciembre de 2014). Por tanto, la muestra la conformaron nueve madres guambianas que, de acuerdo con tipo de estudio, fueron informantes claves. Adicionalmente, como informantes generales, participaron un médico tradicional y tres parteras (dos de ellas médicos tradicionales). Se realizaron seis entrevistas grupales cuando las informantes eran las madres de los niños menores de un año y tres entrevistas individuales tanto al médico tradicional como a las dos parteras de la comunidad guambiana. Las entrevistas grupales permitieron que las participantes expresaran su opinión de forma 
colectiva, lo que facilitó captar su sentir, pensar y experiencias, provocando auto explicaciones que, por la idiosincrasia de las mujeres guambianas, hubiera sido difícil obtener de manera individual. Dado que no todos los participantes hablaban español, el resguardo de Guambía asignó un intérprete de la lengua Misak para poder realizar las entrevistas. Se utilizó el criterio de saturación de datos para determinar el momento en que se debía suspender la recolección de la información. Algunos temas abordados en las entrevistas fueron:

- ¿Usted cómo identifica que su hijo menor de un año está enfermo de los pulmones o del estómago?

- ¿Qué es lo primero que usted hace cuando uno de sus hijos menores de un año presenta enfermedad pulmonar y/o estomacal?

- ¿Cuáles son los principales cuidados que usted brinda a sus hijos menores de un año con enfermedad pulmonar y/o estomacal, para restablecer su salud?

- ¿ ¿Dónde acude usted, si no puede resolver el problema de salud de su hijo menor de un año con enfermedad pulmonar y/o estomacal (dominios)?

- ¿Cómo previene usted la aparición de las enfermedades pulmonares y/o estomacales en los niños menores de un año de su comunidad?

\section{Resultados}

\section{Dominio 1: El susto}

La cultura guambiana es milenaria y ha perdurado a través del tiempo con sus saberes y prácticas propios en torno a la medicina tradicional para curar las enfermedades que afectan a sus integrantes. Esta cultura describe la salud como un estado de equilibrio obtenido al vivir en armonía con los espíritus de los tres espacios: aire, suelo y subsuelo. Cuando se ve afectado el equilibrio en alguno de estos espacios, sobreviene la enfermedad.

Dentro de esta cultura, se plantea que se debe conocer a fondo el espíritu y la energía del niño o niña para poder prevenir que enferme y muera. De hecho, se considera que el olvido de los saberes y las prácticas propias del pueblo $\mathrm{Mi}$ sak, incluyendo la pérdida de los saberes transmitidos por tradición oral por parte de los abuelos, ha generado más enfermedad e incluso la muerte en los niños guambianos. Por este motivo, al indagar sobre las enfermedades que más aquejan a los niños y niñas de esta cultura, encontramos, en forma reiterada, entidades como el susto, el mal de ojo, diarreas, problemas en los pulmones y problemas del estómago.

En relación con el susto, encontramos que, dentro de la cultura Guambiana, se hace referencia a una entidad fantástica atribuida al espíritu de una persona que va a morir, el cual aprovecha el cuerpo de los niños debido a que es débil y le causa esta enfermedad. Los signos y síntomas que lo caracterizan son: vómito, diarrea, ojos hundidos o redondos (si el susto es provocado por un golpe) y variaciones en el pulso (débil, saltón o imperceptible).

El susto tiene diversas causas, la más común son los espíritus en agonía. Así lo ratifican distintas declaraciones de varios de los participantes en el estudio: 
"Lo del susto es cuando una persona va a morir, entonces por visión aparece al niño y como ella va a morir, el niño se asusta y de ahí él llora, le da diarrea, le da vómito, entonces ya uno por ejemplo como médico tradicional tiene su experiencia y trata al niño con la medicina propia” (sic).

"Porque acá en la comunidad Misak tenemos ese conocimiento de que cuando un niño se asusta sale diarrea o sale vómito, o el espíritu de una persona que está a punto de fallecer pega al espíritu del niño y lo asusta, entonces surge la diarrea, el vómito y entonces viene la deshidratación. En el hospital no se dan cuenta qué es lo que tiene el niño, no saben que el niño está es asustado y ellos solo miran que tiene diarrea solamente y le dan suero oral, todas esas cosas, pero dicen los papás de los niños enfermos que llevan al hospital que no se curan"(sic.).

Para determinar si un niño está asustado, la mayoría de los miembros de la cultura indígena guambiana acuden al médico tradicional también. Él puede establecer si el niño verdaderamente está asustado. Dadas las condiciones que anteceden y dependiendo de los signos y síntomas, el médico tradicional utiliza diferentes métodos para determinar si es susto, apoyándose en formas de diagnosticar esta entidad dentro de su cultura y de acuerdo a sus saberes.

El pulseo, es una de las prácticas más utilizadas para determinar los diferentes tipos de enfermedad de los Misak. Cuando se trata de determinar si es susto, el pulso se siente débil o saltón, que se define como el pulso fuerte pero asimétrico; incluso afirman que en algunas ocasiones no se siente. El médico pulseador es el que analiza los latidos del pulso. Sobre el particular, el medico tradicional refirió:

"Pues hay que nivelar el pulso, porque si el pulso está así, no salta así lo normal en un mismo sitio, sino que empieza a ocupar todos los lados, entonces uno tiene que nivelar el pulso, de ahí el tratamiento ya es con plantas. Hay unas plantas que son especiales para eso, la alegría y la orejuela, algunos pulseadores a veces utilizan el tabaco o el cigarrillo" (sic.).

Existen diferentes maneras de curar el susto, de acuerdo con los saberes y dones de cada médico tradicional, se denominan sentidores, oidores o videntes. Una forma de curar el susto es mediante el uso de plantas medicinales. Las más usadas son las plantas calientes como la alegría y la orejuela:

"Sí, yo siempre utilizo esas dos plantas: la alegría y la orejuela, son fundamentales por que la orejuela cumple la función de la armonía y la alegría, esa plantica es la que permite que el niño esté siempre con las energías bien puestas. Sin embargo, hay que tener en cuenta que hay muchas plantas".

De esta manera, si el susto no es tratado y curado, tiene múltiples consecuencias que van a afectar permanentemente la salud y el desarrollo del niño y que incluso pueden causarle la muerte. Uno de los principales efectos es que el espíritu de la persona que va a morir, aleja el cuerpo del niño, o sea, lo divide, anulándolo y 
haciendo que el cuerpo físico del niño enferme. Esto se manifiesta con signos y síntomas como llanto, vómito, mantener los ojos cerrados, los ojos hundidos, entre otros y así lo expresan:

"El susto ha matado a niños cuando no han alcanzado a llegar al médico tradicional, sobre todo cuando el espíritu se asoma en la boca, o cuando se asoma y entra en el cuerpo del niño; cuando se asoma solo por acá no más así en la frente no más, entonces solo da diarrea y pasa rápido. Y cuando es asomado bien en la boca y besa al niño o entra en el cuerpito, entonces eso el niño muere, se deshidrata rápidamente por el vómito y la diarrea, $y$ entonces eso hay que curarle eso rápidamente, si se ve que es eso así o si no el niño muere".

\section{Dominio 2: El mal de ojo}

Dentro de la investigación realizada, se describe una entidad denominada mal de ojo, que es también una dolencia específica en los niños menores de un año de la comunidad misak. El mal de ojo es es una creencia popular, según la cual una persona tiene la capacidad de producir mal a otra persona solo con mirarla. Así, de la persona afectada, en este caso los niños Misak, se dice que "está ojeada, o que le echaron mal de ojo”. Según la cultura misak, este mal se puede producir cuando un niño se lleva a cualquier parte y alguien lo mira, $y$ cuando el niño llama su atención, le causa admiración o envidia por su gracia o su belleza. Entonces, el ojeador se irrita. Es en este momento cuando el ojeador le provoca el mal de ojo. Así lo describió una madre guambiana:
"Cuando hay un niño indígena que es, digamos, bonito, que es avispado y todo, entonces hay personas que les gusta ese niño y si a esa persona le gusta demasiado, entonces ella con el ojo le puede hacer daño, lo enferma, el niño queda ojeado $y$ se sabe porque se mira que el niño queda con un pie más corto y el ojito también, uno es más pequeñito que el otro".

Posteriormente, el niño enferma con el mal de ojo y aparecen varios signos y síntomas. Dentro de los principales se describe, por ejemplo, que el niño afectado presente cuadros de vómito o de diarrea color verde.

Los síntomas físicos que delatan al mal de ojo son, en el caso de los más pequeños, llanto incontrolable y sin causa aparente, dolores de estómago, diarreas, vómitos, brotes en la piel y pesadillas, además de una pérdida de apetito. Y lo más diciente para este mal es que el niño presente un cuadro de tristeza progresivo que no se quita con juegos ni con mimos. Una de las participantes del estudio así lo define:

"Tristeza, eso es lo que mira uno, uno mira la carita del niño y tiene tristeza y uno mira si no tiene otra enfermedad, entonces es porque está ojeado, es cuando uno manda hacer el remedio con una persona que sepa y entonces ya vuelve a la normalidad".

Para saber que un niño está siendo afectado por el mal de ojo, el médico tradicional se guía por diferentes señales. Una de las principales, y quizá la más certera, es que el médico tradicional parpadea involuntariamente tan pronto 
mira al niño afectado. Esto le indica que el mal entró desde los ojos del ojeador hacia los ojos del niño, hasta llegar a afectar su espíritu y enfermarlo.

El uso de remedios fabricados por el médico tradicional, que son para tomar o para aplicarlos en emplastos o infusiones, entre otras formas, es lo que cura al niño y lo libera del mal de ojo. Frente a la medicina tradicional aún hay un gran misterio, puesto que solo la manejan los médicos tradicionales y ellos no revelan todos sus saberes. Afirman que la medicina tradicional es sagrada y que solo puede ser revelada a las personas que nacen con el don de ser, al igual que ellos, médicos tradicionales. Incluso las mismas madres de la comunidad misak no saben qué plantas se utilizan para cada dolencia.

\section{Dominio 3: Prevención de las enferme- dades pulmonares y gastrointestinales en los niños y niñas misak: un conjun- to de saberes propios y milenarios}

La prevención en salud se entiende como la preparación con antelación de lo necesario para un fin, es decir, anticiparse a una dificultad y/o prever un daño. En este caso, se trata de evitar la aparición de enfermedades. El objetivo de la prevención es lograr que un perjuicio eventual no se concrete. La prevención se utiliza en el sector salud como una estrategia a nivel comunitario para lograr mantener un equilibrio en la salud de la población y disminuir los riesgos de que las personas enfermen. Desde esta perspectiva, para evitar la aparición de enfermedades pulmonares y gastrointestinales en los niños y niñas de esta comunidad existen múltiples alternati- vas, que se toman como estilos de vida dentro del pueblo guambiano. Varias de estas estrategias son totalmente válidas dentro de la medicina occidental. Entre ellas tenemos:

- Que los niños estén bien alimentados desde la matriz: esto hace referencia a los múltiples cuidados que la madre debe tener durante su proceso de gestación en cuanto a los alimentos que ingiere. De este modo, la madre mantiene una adecuada nutrición tanto para ella como para el niño que está por nacer y evita múltiples complicaciones y efectos adversos después del parto, garantizando una vida libre de enfermedades en los niños guambianos.

Para lograr lo anterior, una de las prácticas más arraigadas dentro de la comunidad guambiana es, justamente, fomentar y mantener dentro de las parcelas los cultivos propios, tales como hortalizas, legumbres $y$, sobre todo, plantas aromáticas y medicinales, que se caracterizan porque para su cultivo no se utilizan fumigantes, abonos, ni sustancias químicas. Se cultivan maíz, coles, habas, alverjas, ullucos, quinua, papa, oca, arracacha, mahuja. Igualmente, varias plantas medicinales como poleo, higuillo, prontoalivio, manzanilla, malva lisa, malva de olor, descancel, toronjil, borraja, romero, mejorana, cedrón, papayuelo, guayabilla, verbena, limón, lengua de vaca, tay, ajo, churquillo, paico, palo de la cruz, ruda, caléndula, rosas, raíz de quicuy, cope, coca, entre otras. Según una de las madres participantes: 
"Si los niños fueran todos bien alimentados desde la matriz y al seno materno, ellos vinieran todos sanos, porque para nosotros esa es la mejor vacuna. Nosotros no necesitábamos de vacunas antes y vivíamos muchos más años, no como la gente de ahora que se enferma a cada rato y los niños cada vez más desnutridos y más enfermos".

- Administrar leche materna desde el nacimiento hasta completar la edad de dos años: es una de las estrategias de mayor arraigo dentro de la comunidad misak, descritas por la totalidad de las participantes del estudio. Es un factor protector de gran valor y de mayor evidencia científica descrita ampliamente dentro del sector salud. Una de las madres guambianas expresó estas ideas de la siguiente manera:

"Pues yo estoy de acuerdo que se le dé el pecho al niño hasta los dos años $y$ así la mujer no tenga enseguida otro niño. Por eso hay que seguirles dando de mamar hasta los dos años. Evita los siguientes embarazos, porque esos embarazos enseguida es lo que va contra la mamá y el niño, porque la mamá apenas va dando nueve mesecitos leche y ya viene otro $y$ otro y entonces la mamá se desnutre, se le desnutre la matriz, se le desnutre el cuerpo, entonces ahí estamos fregados".

- Evitar que el niño se exponga al frío o el calor extremos: esta es otra forma de prevenir las enfermedades $y$, más específicamente, las enfermedades pulmonares. Se debe recordar que para esta cultura el sistema o dualidad caliente - frío, cobra gran importancia y por lo tanto los niños de esta cultura siempre deben estar protegidos del frío, debido a que la exposición a este hace que el niño entre en un desequilibrio y la enfermedad sobrevenga.

\section{Dominio 4: el poder curativo de las} plantas, una manera de asegurar la salud de los niños en Guambía

En el estudio se describe el uso de las plantas, pues en la cultura guambiana, como en otras culturas indígenas, se usan las plantas como práctica medicinal para la prevención y curación de ciertas enfermedades. Estas comunidades las han utilizado tradicionalmente, acumulando prácticas ancestrales de selección, manejo y conservación de conocimientos que han transmitido de generación a generación.

Igualmente, las plantas medicinales tienen diferentes usos en esta cultura, algunas, en la realización de rituales de ofrecimiento que se hacen a los espíritus cada mes. Son plantas que los indígenas consideran especiales y fundamentales y que cultivan en sus huertas. En esta cultura, los médicos tradicionales son las personas que tienen mayor conocimiento y experiencia en el uso de las plantas medicinales; saben cómo prepararlas, la cantidad de hierbas a utilizar y cómo dárselas a los niños, porque se pueden usar para diferentes enfermedades según la cantidad de planta, y si se usan en exceso, puede ser peligroso para la salud. Así lo explicó el médico tradicional entrevistado: 
"La cantidad de plantas es de acuerdo a la edad, y no solo eso, sino que nosotros miramos es el pulso, si el pulso está débil pues hay que meterle más dosis, si el pulso está más o menos, entonces así se maneja, por lo menos la mayoría de los médicos tradicionales manejan eso o el sentido, entonces el médico tradicional conecta la planta con el niño, si esta planta llega bien al espíritu del niño entonces se siente mejor".

En la prevención, los indígenas guambianos consideran que las plantas medicinales tienen ese poder de evitar que aparezca la enfermedad en los niños, e incluso su efecto es mejor que el de las vacunas. El médico tradicional refiere sobre el particular que:

“...esas plantas también cumplen esa misma función que las vacunas, hacen lo mismo, hay muchas plantas que hacen la misma función o más mejor, porque todas las vacunas sacan de una enfermedad, pero mientras que nuestras medicinas, nuestras plantas, son tan naturales".

Una madre participante también afirmó:

"Hay mucha gente misak que ha usado solo plantas, sin necesidad de vacunar $y$ ha sobrevivido, ya que las plantas también cumplen esa misma función. Las plantas cumplen la misma función que las vacunas o aún mejor porque las vacunas no son tan naturales, las plantas son muy naturales y hacen que cada individuo bien sea niño o niña, sea sano muy sano".

En esta cultura, las plantas cobran gran importancia debido a su significado y al blo misak todo lo necesario para su supervivencia. Las plantas son para ellos la extensión de la madre tierra, parte vital de la vida y de todos sus procesos, entre los cuales se encuentra el crecimiento y el desarrollo de los niños y niñas. Sin embargo, no se debe olvidar que esta etapa es solo una parte de todo el ciclo vital. Por este motivo, los misak siempre reiteran que todo está conectado, así que no se puede dejar de lado los procesos que anteceden el crecimiento y desarrollo de los niños, es decir, la fertilidad, el embarazo, el parto, el posparto. Y tampoco se puede desligar de las etapas que se dan posteriormente a la niñez, es decir, la adolescencia, el adulto joven, adulto y adulto mayor.

De acuerdo con este concepto, se pone de manifiesto que las plantas son parte fundamental para la alimentación, para la protección y para evitar la llegada de enfermedades; en el caso de que algún miembro de la comunidad ya esté enfermo, son también las plantas la principal fuente de cura para los males que aquejan al misak. Los encargados de manejar las plantas son, en su mayoría, las parteras, que generalmente son mamás indígenas, y los médicos tradicionales.

\section{Dominio 5: Manejo energético del niño}

Para los miembros de la cultura misak, el manejo energético del niño hace referencia a todo lo relacionado con el impacto de ciertos acontecimientos decisivos para su vida, desde su nacimiento e incluso antes de la misma concepción. La concepción y el nacimiento están ligados a un acontecimiento esperado, que 
se denomina en su lengua la "llegada". El nacimiento de un niño o niña dentro de esta cultura, al igual que el embarazo, se encuentran profundamente relacionados con el mundo sobrenatural y, en general, con su concepción de la naturaleza y del organismo humano. Por estas razones, consideran importante determinar, según la fecha de nacimiento, el tiempo de "llegada" del niño o niña misak, lo cual se define principalmente por las fases de la luna. Lo que resulte va a establecer la fortaleza del nuevo ser o su debilidad, ya que, dependiendo en qué tiempo lunar haya nacido el niño o niña, así mismo será su energía: puede verse afectado positivamente, en mayor o menor medida, o puede ser débil y de poca energía. Así lo expresa un participante del estudio:

"nosotros no decimos que nació, sino que llegó, así, si el niño llega en luna llena, así se comporta, puede ser muy fuerte o débil. Entonces ese es el manejo energético con los espíritus naturales, pero eso se va perdiendo, ya no se valora ese conocimiento que nosotros como pueblo misak tenemos".

En tanto el niño o niña misak se van a ver afectados desde lo que antecede al parto, se habla entonces de la gestación, e incluso de la fecundación, debido a que todo viene entrelazado y hace parte del ciclo de vida del misak. La gestación está íntimamente ligada a este acontecimiento (la llegada) y es por ello que si, desde el mismo momento de la fecundación, la mujer no se cuida, sobrevienen las consecuencias negativas para la llegada o nacimiento del niño, y también para la madre. Una mujer en estado de embarazo debe cuidarse adecuadamente, pues de sus cuidados dependerá mu- cho la salud y bienestar futuros, tanto de ella como del niño o niña que va a nacer, o como ellos lo denominan, el que va a "llegar".

\section{Dominio 6: Causas de enfermedad y muerte en los niños misak}

Los guambiamos no conciben una buena salud si no se vive en armonía con la naturaleza; en esta relación son sagrados los bosques, los picos, los cerros, las montañas y las lagunas. Los ríos y las quebradas se relacionan con las venas y las arterias que corren por el cuerpo humano. La salud se pierde cuando se desequilibra la relación con la naturaleza, con el espíritu, con el cuerpo y con los mayores, porque ya no se escucha el consejo, sino que se mantiene en una permanente búsqueda de lo material(8). Una de las participantes lo plantea así:

"Para nosotros la salud es algo tan natural; si uno vive en armonía y en equilibrio con la naturaleza, anda con buena salud, pero si uno anda en desorden $y$ desequilibrio con la madre tierra, entonces aparecen las enfermedades. Tenemos los espíritus del territorio, los espíritus del suelo y del subsuelo, de los tres espacios, si no cumplimos las normas naturales nos atacan, nos atacan y nos causan enfermedad".

Es decir, consideran que, al perder el equilibrio entre lo espiritual y territorial, aparece la enfermedad, que se da por falta de conexión con el territorio, las plantas medicinales y la madre tierra. Otra causa de enfermedad es no cumplir las reglas o las normas naturales, por lo tanto, los espíritus del te- 
rritorio, del subsuelo y del espacio, atacan. Uno de los médicos tradicionales expresó:

“...antes los misak no nos sentíamos enfermos, pues relacionábamos muy bien con todos esos saberes naturales como pueblo misak, pero hoy en día, hemos olvidado esa cultura tan natural que hemos tenido y también llegan las enfermedades porque estamos desequilibrados espiritual, moral y territorialmente, no estamos conectados con el territorio, no estamos conectados con las plantas medicinales $y$ por eso las enfermedades".

Según la cosmovisión de esta cultura, las enfermedades pueden tener origen en el frío y el calor. Las enfermedades que tiene origen en el calor pueden ser la fiebre, que se produce cuando hierve la sangre en el cuerpo, producto de un susto, por ejemplo. Las enfermedades que tienen origen en lo frío causan problemas pulmonares, asociadas básicamente a la exposición de los niños al sereno, al viento, al frío, o al humo del pino verde o pino extranjero. La gripe y el resfriado se atribuyen al espíritu de la gripe.

Estas enfermedades no se deben tratar con medicamentos farmacéuticos, sino con plantas medicinales. El "estado de contaminación" es un elemento clave dentro del concepto de salud que tienen los Misak. Se encuentra asociado a lo "frío y sucio" y su fuente principal es la mujer quien, con su sangre menstrual, "fría, sucia y peligrosa", o con la sangre del parto, se contamina y puede llegar a contaminar a otras personas, cosas y lugares. De la misma manera, los espíritus de los muertos tienen esta capacidad. El "estado de contaminación" es la principal causa de irritabilidad de los espíritus y duendes, quienes pueden reaccionar matando o enfermando a las personas. Hace que los niños se pierdan en el monte o los matan, rompen las herramientas, despeñan los animales, secan los cultivos, etc.(9). Uno de los participantes afirmó:

“...porque acá el periodo de la menstruación de la mujer hay que respetarlo, porque en ese momento de la enfermedad no debe salirse a trabajar, ni salir al campo porque se está violando esas normas naturales. Pero si se guarda los cuatro días de ese periodo en casa y no se acerca a los ojos de agua, no se presentan enfermedades".

Las causas más frecuentes de los problemas respiratorios en los niños y niñas misak tiene origen en la exposición al frío y al espíritu de la gripa. Cuando se desencadenan los problemas respiratorios, la sintomatología descrita desde los saberes de la cultura Misak va desde la aparición de la fiebre, la tos, el "moqueo" (rinorrea), el enfriamiento de la molleja, hasta la falta de sueño y el llanto excesivo.

Uno de los aspectos que se debe tener en cuenta para esto y que es imprescindible, son los cuidados básicos del recién nacido. Si no se realizan según sus costumbres, los niños pueden enfermar. Esta cultura tiene como práctica que ningún recién nacido se bañe inmediatamente, sino que lo protejan del frío para evitar la hipotermia, así como cuidar los ojos y los oídos del viento porque consideran que, al haber pérdida del equilibrio 
caliente - frío, el niño o niña puede enfermar. Consideran que estos cuidados se brindan cuando los niños nacen en la casa o permanecen en la comunidad. Por el contrario, no se practican cuando el parto ocurre en un centro de salud o cuando el bebé se lleva a un centro hospitalario. Ante esto, dos médicas tradicionales afirmaron:

"cuando el recién nacido nace en el hospital se tiene que prevenir de toda enfermedad, lo que es el frio, lo que es la visión, no le cuidan los ojitos, el oído.... Porque cuando uno tiene el parto en caso, entonces nuestras mayores nos encierran en un cuarto, nos cobijan bien, tapan al niño y no lo dejan ver hasta el mes, no lo dejan salir a ventiar por el riesgo de enfermar por consecuencia del frio, contrario a lo que pasa al hospital.

\section{Dominio 7: La brecha entre el saber científico y el saber cultural, una de las causas de olvido de la medicina propia}

La transmisión de conocimientos culturales en la comunidad guambiana, así como en otras culturas, se hace de generación en generación por medio de la comunicación oral, con el fin de garantizar que las prácticas y saberes se conserven y la cultura se preserve en el tiempo y en el espacio. Los mayores (ancianos), también llamados taitas y mamas, son las personas con gran conocimiento cultural que poseen la autoridad para tomar las decisiones. Comunican y comparten sus conocimientos a sus hijos y nietos alrededor del Nachak o fogón, un espacio común donde se construye la familia, se comparte un diálogo y se realiza una trasmisión de saberes. Aquí también se realizan los rituales y se convierten en espacios cotidianos de aprendizaje del lenguaje natural del cosmos, que contribuyen a la formación integral del misak(9).

Una de las madres del estudio manifestó durante la entrevista:

"Siempre los mayores le dan esos conocimientos a uno alrededor del fogón y hablaban de las creencias que teníamos que seguir; esos conocimientos que nos dan es la sabiduría propia que hay que mantener y tener en cuenta".

"Nuestras abuelas y madres enseñaban, comunicaban oralmente cómo debemos cuidar desde nuestros orígenes, desde nuestra cultura, el cuidado que debemos tener...las mujeres de la cultura guambiana tienen mucha ciencia, mucha experiencia, son las que cuidan y tienen los saberes".

En el caso de la salud, la medicina tradicional se ha ido deteriorando. Por esta razón, en 1991, el Cabildo de Guambía logró el primer objetivo mediante el proyecto de plantas medicinales en Santiago de Guambía, que estuvo conformado por un vivero y por la dotación de la casa de Sierra Morena, contribuyendo al fortalecimiento de la medicina tradicional. A pesar de que el diagnóstico en el plan de vida del pueblo Guambiano incluye como proyecto fundamental el fortalecimiento de la medicina propia basada en las plantas y la cosmovisión misma de la comunidad, dentro del actual programa de salud se ha fortalecido la medicina occidental, desplazando o 
aislando totalmente los valores ancestrales de promoción y prevención; las plantas medicinales se toman como una medicina alternativa.

Así pues, los indígenas guambianos consideran que la salud de la comunidad está dirigida totalmente por los entes gubernamentales, quienes no tienen respeto hacia las culturas ancestrales porque quieren imponer la medicina occidental por medio del cumplimiento a los programas de salud, las leyes y reformas de la salud del Ministerio de salud y de la protección social. Un participante lo expresó durante la entrevista de la siguiente manera:

“...llega el programa de salud desde la ley 100 y ahí nos exigen metas, compromisos $y$ cantidad de vainas que no concuerdan con nuestra realidad como pueblo misak, entonces ahí nos chocamos con ellos... nosotros queremos intentar hacer desde la visión de la salud que tenemos, pero no nos entienden, nos obligan a hacer esos controles desde la cultura occidental, desvalorando todos los saberes propios, entonces tenemos esa dificultad".

Otro factor que se considera que ha aumentado la brecha entre el saber científico y el saber cultural tiene que ver con el personal de salud que atiende en el hospital de Mama Dominga, pues no se informa al Cabildo de Guambía sobre la contratación de nuevo personal. Además, al personal médico nuevo contratado no se le realiza la socialización de la parte cultural antes de iniciar su labor con la comunidad, ni la respectiva inducción y empalme de los procesos que se están adelantando entre la medicina propia y la medicina occidental. Esta es la descripción de un participante sobre este asunto:

"Cuando hay cambio de médico rural, entonces vienen con una mentalidad de afuera y pues yo le decía al Cabildo que al contratar esos médicos era socializarlos primero y decirles cómo es la parte cultural y los programas que se están trabajando para que ellos tuvieran conocimiento $y$ continúen... pero a veces el coordinador de Mama Dominga llegaba y contrataba el personal y a veces el Cabildo no se daba cuenta, entonces llegaban con una mentalidad de afuera que era tenaz uno compartir".

\section{Temas}

1: El equilibrio entre las fuerzas energéticas de los misak, una búsqueda permanente para la prevención de enfermedades

De acuerdo con los hallazgos de la investigación, se debe resaltar la importancia de ciertas prácticas de cuidado de los misak, que van enfocadas a mantener el estado de bienestar y equilibrio, en pro de mantener la salud.

Para los miembros de esta cultura no existe un estado de salud sino de equilibrio, como ya se describió. El modo en que las madres abordan estas prácticas de cuidado para sus hijos va encaminados a la protección y a la prevención. Así como la alimentación basada en los cultivos propios para ellos cobra importancia y sirve de método de enseñanza del amor y respeto por la madre tierra, 
también cobran importancia otro tipo de prácticas de cuidado, por ejemplo, la lactancia materna como fuente principal de alimentación durante los primeros 6 meses de vida y luego de forma complementaria hasta los 2 años de vida. Las madres parten de la premisa de que la naturaleza es sabia y que el pecho se hizo para proveer el alimento para sus hijos, al igual que lo hacen el resto de especies de mamíferos en la tierra.

El uso de plantas medicinales también aporta en gran medida al cuidado de los niños y niñas de esta cultura como método de protección y prevención de enfermedades y para armonizar la relación de los pequeños con la pacha mama $y$ todo lo que en ella habita. Mantener la espiritualidad como forma de prevención de enfermedades, agradar a los espíritus de la madre tierra y mantener un equilibrio constante, son otras de las maneras como los misak perciben su estado de buena salud o evitar la aparición de enfermedades.

2: El respeto a la cultura y el diálogo como fundamento de una relación asertiva entre saberes

Es reiterada y contundente la percepción de la comunidad guambiana acerca del desconocimiento de su cultura por parte del personal de salud, y del atropello que representa la imposición de métodos, procedimientos y prescripciones de la medicina convencional. Sin embargo, más allá de estos sustentados reclamos, es evidente que la negación y resistencia frente al sistema de salud no es total, que se considera y aplica como opción cuando lo culturalmente concebido agota los recursos y no logra solucionar los problemas presentados; lamentablemente, esto suele suceder demasiado tarde; además, no es general, por lo cual, como ya se planteó, las cifras de morbilidad y mortalidad se mantienen altas en relación con las metas de la salud pública, en este caso, especialmente, respecto a las dos patologías infantiles abordadas y para la población menor de un año.

El reconocimiento del otro como sujeto y de su cultura como contexto se ratifica, pues como sustancial a los procesos, se hace indispensable asumir y promover entre el personal de salud. Pero a un verdadero reconocer le antecede necesariamente un conocer y un interactuar, asumiendo al otro como un igual en términos de la condición de ser humano. Debe propiciarse que se conozcan y valoren los argumentos y sustratos de cosmovisión, guambiana o misak, y que dicho conocimiento y valoración permita concretar formas de diálogo con los sujetos cuidadores y con la comunidad en general. Deben hacerse en relación con la necesidad de atender y proteger la población infantil menor de un año, pudiendo, dentro de lo posible, además de reconocer al otro en lo suyo, tratar de acercarlos al reconocimiento del saber formal como una opción capaz de aportar soluciones concretas.

\section{Discusión}

Para el misak, nacer, enfermar y morir, entre otros eventos de la vida, son fundamentales para el desarrollo de su pueblo, ya que, además de mostrar la dinámica de la existencia biológica y cultural del grupo, se convierten en indicadores del tipo de relación existente entre los 
individuos y su medio. Las condiciones necesarias para estar sanos o para estar enfermos circulan por todos los espacios donde vive el guambiano. Si una persona no quiere enfermar, debe ser muy cuidadosa y estar pendiente para evitar las condiciones que pueden afectarla. Por ello, se deben guardar normas relacionadas con el comportamiento entre los individuos de la misma cultura y con los de otras culturas, con el medio ambiente, con lo económico, con lo político, etc., es decir, deben comportarse según las costumbres propias del pueblo guambiano (10).

Es claro, entonces, que la población indígena guambiana posee desde tiempos ancestrales su propio sistema de salud y que los médicos tradicionales lo practican, ya que son conocedores de la naturaleza espiritual y física. Según su propia percepción, desde la llegada de los "conquistadores" o "invasores", el sistema de salud propio ha querido ser desplazado por la llamada medicina occidental. Sin embargo, hoy persiste todavía, en contra de los diferentes intentos que se han hecho a través de la historia por acabarlo, especialmente los "sistemas de mercado del sistema de salud occidental" y las sectas religiosas, que quieren marginar a los médicos tradicionales, especialmente, ya que han venido perdiendo cobertura y credibilidad (11).

Desde esta perspectiva, se debe entender que la mayoría de las prácticas de cuidado que la comunidad asume desde la propia visión cultural, van en pro del mantenimiento de la salud en general y

específicamente la salud de los niños y niñas guambianos, ya que están enca- minadas a mantener el equilibrio y la buena relación del misak con la madre naturaleza y todo lo que en ella habita. Varias de las prácticas descritas dentro del presente estudio favorecen la preservación de la salud y se podrían catalogar como prácticas de estilos de vida saludable y prácticas de promoción y prevención. Otras son inocuas y muy pocas afectan negativamente la salud del misak. Por este motivo, es importante tratar de entender mejor por qué dentro de esta cultura se realizan ciertos rituales, por qué es tan importante el uso de la medicina propia y por qué no se hace uso de la medicina ortodoxa.

Es fundamental reconocer que los cuidados culturales son tan válidos como los saberes occidentales; ambos encierran gran valor y por lo mismo no se debe anteponer uno sobre otro, sino más bien aunar esfuerzos para preservar los cuidados que responden a prácticas preventivas o protectoras y negociar algunas que, sin necesidad de erradicarse, pueden continuar haciendo parte del legado cultural de los misak con mínimo o ningún impacto negativo sobre la salud de sus miembros.

Dentro del estudio realizado, tal como ya se había mencionado, se encontraron cuidados y prácticas que deben ser preservados, debido a que van en beneficio de la salud, no solo de la niñez sino de la comunidad misak en general, como lo son la alimentación propia y la lactancia Materna.

Sin embargo, hay ciertas prácticas que se deben negociar, puesto que, si bien no causan la muerte en los niños de esta cultura, incrementan la morbilidad. Por 
ejemplo, se hace referencia al uso de emplastos calientes en niños que presentan problemas gastrointestinales, debido a que muchas de las plantas utilizadas en los emplastos liberan sustancias que, al estar en contacto con la piel por largo tiempo, pueden producir irritación, laceración e incluso infecciones locales. Esto puede causar hospitalizaciones prolongadas, uso de antibióticos y demás problemas derivados de este tipo de prácticas propias dentro de la cultura misak. Por lo tanto, se deben explicar los riesgos a los que se expone el niño, para así acordar disminuir esta práctica en cuanto al tiempo y a que no se aplique directamente sobre la piel de los niños.

Cabe resaltar que ,la mayor parte del personal que labora en el hospital Mama Dominga (hospital propio de dicha comunidad), pertenece a la cultura misak, lo que minimiza los obstáculos o barreras relacionadas con la comunicación y mejor la relación enfermera-paciente.

\section{Conclusiones y recomendaciones}

La cultura guambiana considera que la enfermedad se origina en una pérdida de la armonía, conexión y relación de la persona con la naturaleza, con el espíritu y con su cuerpo, generalmente producida por el desequilibrio en la relación fisiológica caliente/frío. En consecuencia, los indígenas adoptan estilos de vida y costumbres en pro de la preservación de dicho equilibrio y, en general, del equilibrio entre el individuo y el ambiente que lo rodea. Esto explica el porqué de, cuando los niños presentan problemas de sa- lud, las madres de los menores adoptan medidas para restablecer el equilibrio térmico dentro del cuerpo y del ambiente. De este modo, responden a sus necesidades de forma inmediata y, la mayoría de las veces, de forma acertada.

Para ser competente culturalmente, el profesional de enfermería debe trabajar teniendo en cuenta la cosmovisión de esta comunidad, pues solo de esta manera podrá lograr que se preserven y se mantengan los cuidados culturales que brindan las madres de la cultura guambiana y que favorecen realmente la salud en los niños. Una gestión de cuidados de enfermería culturalmente congruente y competente con la cultura permite la comprensión de los valores, prácticas y saberes de las diferentes culturas en las que actúa, lo cual es fundamental para consolidar una adecuada práctica en enfermería.

Para el personal de salud que interviene en el contexto de la cultura misak, es importante e ineludible estudiar del sistema médico guambiano, así como evidenciar y comprender sus concepciones sobre salud y enfermedad, sus prácticas preventivas, curativas y recursos médicos, los cuales se fundamentan en la cosmovisión que norma las actividades sociales y culturales de esta comunidad y que constituyen valores ancestrales que los ayudan y conducen a estar en armonía con el universo y con todas las manifestaciones que ofrece la vida.

Los enfermeros y enfermeras están llamados a crear espacios que consideren los saberes y prácticas propias de esta cultura para lograr que la comunidad 
sus derechos, sus saberes y sus prácticas, brindando un cuidado holístico, no fragmentado, donde solo el personal de salud impone sus conocimientos y no tiene en cuenta los conocimientos ancestrales de la cultura misak.

\section{Referencias}

1. Cepeda C., Flórez V., Vásquez M. Prácticas de cuidado del recién nacido: una mirada desde las madres de la cultura guambiana en el departamento Cauca. 2012. [Citado: 2013 Diciembre 13]. Universidad del Valle. Escuela de Enfermería. $12 \mathrm{p}$.

2. Base de datos del Hospital Mama Dominga. Silvia, Cauca: Oficina de estadística, c2012-2015 - [Citado 2014 Enero 22].

3. Teorías de Enfermería [Internet]. El cuidado: Madeleine Leininger; 2012 Jun 11. [Citado: 2014 Abril 8] Disponible en: http://teoriasdeenfermeriauns.blogspot. com/2012/06/madeleine-leininger.html

4. Mejía L., López L. La familia y la cultura: una conexión innovadora para el cuidado de la salud. Index de Enfermería. 2010;19(2-3):138-42.

5. Leininger, M. Culture care diversity and universality: a theory of nursing. Nueva York: Nacional League for Nursing Pres; 1991. LEININGER, Madeleine. EI Cuidado, Artículo biografía de Madeleine Leininger. 2012. [Citado: 8 abril 2014] [On line] <http://teoriasdeenfermeriauns.blogspot. com/2012/06/madeleine-leininger.html

6. BLVD, Wilson. National Alliance on Mental Illness.Star Center Vol 1, (2009). [Citado: 13 diciembre 2013]. [On line]: www.consumerstar.org.

7. ARAGON, Raúl y Sánchez: Enrique Departamento de Planeación Nacional, 1997, [Citado 12 oct. 2013]
8. Revista nuestras raizes: un recorrido por una de las culturas colombianas más conocidas y peculiares, guambianos o misak 2012 [Internet]. Universidad Manuela Beltrán. [Citado 2013 diciembre 13]. Disponible en: <www.slideshare.net/ oscartibaduizar/revista-guambianos Ibid 5 p. TIBABUYES, Oscar. Revista Nuestras Raíces: Un recorrido por una de las culturas colombianas más conocidas y peculiares, guambianos o misak. (2012). [Citado: 13 de diciembre de 2013]. [On line] www.slideshare.net/oscartibaduizar/ revista-guambianos

9. Instituto colombiano de Antropología. Introducción a la Colombia Amerindia. Bogotá: Instituto colombiano de antropología, 1987. Capítulo 17, Guambía; p. 265-83

10. Espitia, Javier. Rodríguez, Luis Carlos. El papel de la Comunicación en las disputas por el Derecho a la Medicina Propia: el caso del pueblo indígena Misak en el departamento de Cauca (Colombia). Capítulo 27. [Citado: 21 de mayo de 2016] 353 a 385p. [On line]: ftp://ftp.unicauca.edu.co/ cuentas/.cuentasbajadas29092009/dtm/ docs/EHAS-AIEPI/CD-Programa-capacitadores/Area-Socio-Antropologica/APROXIMACION-CONCEPTUAL-A-LA-PROBLEMATICA.pdf

11. TAMPU, Portal Ecoturístico y Multicultural del Cauca [Internet]. Popayán. Cauca: Universidad del Cauca. Etnografía, el pueblo guambiano. 2002 diciembre. [Citado: 2016 mayo 21]. Disponible en: http:// tampu.unicauca.edu.co/merlin/preview/ viviendo. php?id=17 


\section{Información}

\section{INSTRUCCIÓN A LOS AUTORES PARA LA PRESENTACIÓN DE ARTICULOS EN ESTA REVISTA}

A continuación se presentan la forma y preparación de los artículos para ser publicados en la revista.

\section{TÍTULO}

\section{AUTOR(ES)}

El orden de mención debe reflejar la magnitud e importancia de la contribución de cada individuo.

\section{INSTITUCIÓN(ES) Y DIRECCIÓN POSTAL}

Debe ir al pie de la primera página.

\section{RESUMEN}

El resumen debe ser estructurado así: Introducción, Métodos, Resultados y Conclusión.

El resumen debe ser máximo de 150 palabras. Los verbos usados se conjugan en tiempo pasado.

\section{PALABRAS CLAVE}

Facilitan las entradas (descriptores) en los sistemas de indización y de recuperación de la información. Por favor apóyese en los tesauros o en el www.bireme. br donde puede consultar el descriptor $\mathrm{DeC} / \mathrm{MeSH}$ y desde allí se le orientará para la definición de las palabras clave y también identificará la traducción de los términos de búsqueda.

\section{INTRODUCCIÓN}

En su elaboración se sugiere mencionar brevemente los siguientes aspectos:

1. El problema o el objetivo, indicando su origen, antecedentes e importancia.

2. Los conocimientos existentes sobre el tema, resumiendo los resultados de las investigaciones anteriores y su referencia bibliográfica.

3. Tipo de diseño utilizado, con una breve sustentación sobre su elección.

4. Definición de las principales variables, términos y abreviaturas.

5. Expresar el tiempo en presente. Un error es la ausencia de respaldo bibliográfico.

\section{MATERIALES Y MÉTODOS}

Detalla la manera como fueron obtenidos los resultados en cuanto al diseño, técnicas y materiales utilizados, para que la investigación pueda replicarse.

EN LOS MATERIALES NO SE OLVIDE INCLUIR

1. Especificaciones técnicas y cantidades exactas.

2. Nombre genérico, químico y comercial.

3. Procedencia, nombre del fabricante. 
1. En caso de trabajar con animales, plantas y microorganismos, identificar género, especie $y$ cepa; características especiales de edad, sexo, condición genética y fisiológica.

2. En el caso de humanos, identificar las características principales de la población y de la muestra.

\section{EN LOS MÉTODOS PROCURE}

1. Expresar el tipo de diseño empleado.

2. Tipo de muestreo utilizado.

3. Mencionar las técnicas estadísticas y de los instrumentos de recolección de información; reportar indicadores de validez interna y externa de las pruebas empleadas sin recurrir a demostraciones; en caso de pertinencia, expresar la referencia bibliográfica.

4. Mencionar aspectos importantes del trabajo de campo.

5. Utilizar los verbos en tiempo pasado.

\section{PARA EL DISEÑO DEL ESTUDIO}

Es importante aclarar cómo fue la selección de sujetos u objetos y la asignación de sujetos u objetos a grupos de estudio (experimental, control, intervención tratamiento). Se debe ser específico acerca de la medición del efecto y de la exposición. Los métodos de análisis deben ser sustentados y acordes a los objetivos del estudio.

\section{RESULTADOS}

Esta parte del artículo tiene por objeto presentar la verdadera contribución del estudio realizado. En esta sección se presenta información pertinente a los objetivos del estudio en forma coherente y además las pruebas estadísticas aplicadas a la información recolectada. No olvide que debe mencionar todos los hallazgos relevantes, incluso aquellos contrarios a la hipótesis, también debe incluir detalles suficientes para justificar las conclusiones.

\section{PRESENTACIÓN DE LOS} RESULTADOS

Aquí el autor(es) puede(n):

1. Utilizar cuadros cuando sea necesario, resumir la información.

2. Cuando se trate de uno o pocos datos, descríbalos en el texto.

3. Las figuras estadísticas, croquis y esquemas ayudan a aclarar los resultados.

4. No debe repetir con palabras la información contenida en los cuadros y figuras, solamente expresar las conclusiones que se deduzcan de ellas.

5. Los datos estadísticos encontrados con pruebas de significancia, deben expresarse con interpretaciones y conclusiones claras, sencillas y concretas.

6. Los verbos en esta sección se recomienda que sean conjugados en tiempo pasado.

\section{REQUISITOS PARA CUADROS Y FIGURAS}

Los títulos de los cuadros van en la parte superior y los de las figuras se escriben en la parte inferior de las mismas.

1. Deben ser autoexplicativos, sencillos y de fácil comprensión.

2. Indicar el lugar, fecha y fuente de origen de la información. 
1. Se deben incluir unidades de medidas relativas (razones, porcentajes, tasas, índices).

\section{DISCUSIÓN}

$\mathrm{Su}$ fin es mostrar las interpretaciones, generalizaciones, relaciones, conclusiones y recomendaciones que los resultados indican y las discrepancias con otros hallazgos encontrados en investigaciones citadas en los Antecedentes y el Marco Teórico.

\section{SUGERENCIAS}

1. Exponga los principios, relaciones y generalizaciones que los resultados indican.

2. Señale las excepciones, la falta de correlación y delimite los aspectos no resueltos.

3. Muestre cómo concuerdan (o no) sus resultados e interpretaciones, con los trabajos anteriormente publicados.

4. Formule las conclusiones de la manera más clara posible.

5. Resuma las pruebas que respaldan cada conclusión.

6. Tiempo pasado para sus conclusiones y presente para las referencias bibliográficas. Las conclusiones son parte de la discusión de resultados y permiten inferir o deducir una verdad de otras que se admiten, demuestran o presuponen.

\section{AGRADECIMIENTOS}

Esta sección reconoce la cooperación de personas e instituciones que ayu- daron materialmente al autor en su investigación.

\section{REFERENCIAS}

Las referencias permiten identificar las fuentes originales; dan solidez a los hechos y opiniones expresadas por el autor. Orientan además al lector para que se informe en mayor extensión. Para ampliar esta información, se le sugiere consultar las Normas Vancouver.

\section{REFERENCIAS PARA UNA REVISTA}

1. Autor(es) apellidos.

2. Título del artículo.

3. Nombre de la revista.

4. Año de la publicación.

5. Número del volumen.

6. Página (primera y última).

\section{REFERENCIAS PARA UN LIBRO}

1. Autor(es) o Editor(es).

2. Título.

3. Edición (si no es la primera).

4. Ciudad, sede de la editorial.

5. Editorial.

6. Año de la publicación.

\section{APÉNDICES O ANEXOS}

Para completar o ilustrar el desarrollo del tema, se debe incluir información que por su extensión o configuración no encuadra bien dentro del cuerpo del artículo.

NOTA: Los autores se someten al proceso de selección y arbitraje de la revista Investigaciones Andina. 